\title{
Myocardial remodeling and bioelectric changes in tachycardia-induced heart failure in dogs
}

\author{
B. Song, B.N. Wang, D.N. Chen and Z.G. Luo
}

Department of Cardiovascular Medicine, The First Affiliated Hospital, Anhui Medical University, HeFei, Anhui Province, China

\begin{abstract}
In this study, electrical and structural remodeling of ventricles was examined in tachycardia-induced heart failure (HF). We studied two groups of weight-matched adult male mongrel dogs: a sham-operated control group $(n=5)$ and a pacing group $(n=5)$ that underwent ventricular pacing at 230 bpm for 3 weeks. Clinical symptoms of congestive HF were observed in both groups. Their hemodynamic parameters were determined and the severity of the HF was evaluated by M-mode echocardiography. Changes in heart morphology were observed by scanning electron and light microscopy. Ventricular action potential duration (APD), as well as the 50 and 90\% APD were measured in both groups. All dogs exhibited clinical symptoms of congestive HF after rapid right ventricular pacing for 3 weeks. These data indicate that rapid, right ventricular pacing produces a useful experimental model of low-output HF in dogs, characterized by biventricular pump dysfunction, biventricular cardiac dilation, and non-ischemic impairment of left ventricular contractility. Electrical and structural myocardial remodeling play an essential role in congestive HF progression, and should thus be prevented.
\end{abstract}

Key words: Tachycardia; Heart failure; Action potential duration

\section{Introduction}

Control of cardiac remodeling is a prerequisite for preventing the development of heart failure (HF) after myocardial infarction (1-3). Ventricular remodeling, first described in animal models with left ventricular stress and injury (1-3), occurs progressively in untreated patients after massive myocardial infarction and in those with dilated forms of cardiomyopathy (1-3). The gross pathologic changes such as increased left ventricular volume and perturbation of the normal elliptical left ventricular chamber configuration are driven at the histological level by myocyte hypertrophy and apoptosis and increased interstitial collagen (1-3). These findings substantiate the importance of ventricular remodeling in the pathophysiology of HF progression and support the role of assessing left ventricular remodeling in the clinical investigation of novel HF treatments (2-9). Myocardial remodeling and bioelectric changes for the progression of $\mathrm{HF}$, however, remain to be elucidated.

The term ventricular remodeling refers to alterations in ventricular architecture, with associated increases in volume and alterations in chamber configuration, driven at the histological level by a combination of pathologic myocyte hypertrophy, myocyte apoptosis, myofibroblast proliferation, and interstitial fibrosis (10). Aside from the effects of abnormal cardiac structure on cardiovascular events, the relationship between left ventricular remodeling and clinical outcomes is likely linked through common local and systemic factors that drive vascular as well as myocardial pathology $(1,4-8)$.

Congestive HF is a serious clinical syndrome with progressive myocardial dysfunction and poor clinical outcomes (1-9). A milestone in HF progression is myocardial remodeling. Changes in ventricular geometry (remodeling) are recognized as a major determinant of the development of impaired ventricular function and poor prognosis (1-9). However, the mechanisms responsible for HF progression and remodeling remain to be elucidated.

Rapid right ventricular pacing in dogs reportedly produces a useful model of low-output HF $(3,8,11-13)$. However, little information is available on the cardiac remodeling changes in animal models of pacing-induced HF. In the present study, the effects of rapid ventricular pacing on cardiac hemodynamics, mass, chamber size, and action potential duration (APD) in dogs were evaluated for 3 weeks.

\section{Material and Methods}

\section{Animal model}

Weight-matched adult male mongrel dogs (22-23 kg) were obtained from the animal laboratory of the Anhui 
Medical University. This study was carried out in strict accordance with the recommendations in the Guide for the Care and Use of Laboratory Animals of the National Institutes of Health. The protocol was approved by the Institutional Animal Care and Use Committee of the First Affiliated Hospital of Anhui Medical University (Permit No. 20060828001). The dogs were randomly assigned to 2 groups: a rapid right ventricular pacing group $(n=5)$ and sham controls $(n=5)$. The dogs were anesthetized with thiamylal sodium (30 mg/kg, iv). Catheters were placed in the right ventricle, and a pacing lead (Medtronic Company, USA) was placed in the apical area and lateral wall of the right ventricle under general anesthesia and with fluoroscopic guidance. A power generator (Medical Electronics Laboratory of Engineering, Department of Fudan University, Shanghai, China) was connected to the pacing leads and inserted into a subcutaneous pocket in the abdomen. Baseline studies were performed 10-14 days following surgical procedures already described (14). Ventricular pacing was then initiated at $230 \pm 10 \mathrm{bpm}$ continuously for 3 weeks under the following conditions: stimulus pulse width $0.15 \pm 0.05 \mathrm{~ms}$, pulse range $500 \Omega$, and threshold voltage $<1 \mathrm{~V}$. The five control dogs underwent the same treatment but without pacing (14).

\section{Hemodynamic echocardiography}

Hemodynamic data were obtained from conscious, unsedated animals $(9,10)$. The dogs were inactivated (that is, pacing was turned off), remaining for at least $1 \mathrm{~h}$ in a basal state after pacemaker implantation $(15,16)$. All data were obtained from each animal after 14 days. Pressures were obtained from the left ventricle. Two-dimensional and M-mode images were obtained using a GE Vivid 7 (2.5-3.5 MHz) imaging system (USA). Images were obtained using a right parasternal approach at the midpapillary muscle level and recorded on VHS tape. Measurements were made according to echocardiographic criteria. Short-axis M-mode measurements were made through the interventricular septum (IVS) and anatomic lateral wall, because of the canine IVS midline orientation and use of the right parasternal view. All parameters were measured on at least five random end-expiratory beats and averaged. The end-diastolic dimensions (EDD) were obtained at the onset of the QRS complex. End-systolic dimension (ESD) was taken at the instant of maximum lateral positioning of the IVS or at the end of the T wave. Left ventricular systolic function was assessed using fractional shortening $=[(E D D-E S D) / E D D] \times 100$. The animals were imaged on 2 consecutive days before initiation of the pacing protocol to demonstrate the reproducibility of echocardiographic measurements. Data from separate determinations were highly reproducible. Left ventricular size and function measurements were obtained from the rapid pacing and control groups at the conclusion of the 3 -week protocol. The dogs were taken to the recording room, where the pacemaker was deactivated. A quiet environment was maintained to avoid stress and, 30-40 min after pacemaker deactivation, data were collected under baseline conditions.

\section{Action potential duration}

The animals were anesthetized after 14 days of pacing, and midline sternotomies were performed. The dogs were anesthetized with thiamylal sodium (30 mg/kg, iv; $1 \mathrm{~mL} / \mathrm{kg}$, iv). The still-beating hearts were immersed in saline $\left(4^{\circ} \mathrm{C}\right)$. Transmural samples from each region were rapidly frozen in liquid nitrogen and stored at $-70^{\circ} \mathrm{C}(10,17)$ for histological analysis.

The heart was perfused at a constant pressure of $80 \mathrm{~cm} \mathrm{H} \mathrm{H}_{2} \mathrm{O}$ with Kreb's solution (117 mM NaCl, $4.7 \mathrm{mM}$ $\mathrm{KCl}, 2.5 \mathrm{mM} \mathrm{CaCl}_{2}, 1.2 \mathrm{mM} \mathrm{NaH}_{2} \mathrm{PO}_{4}, 26 \mathrm{mM} \mathrm{NaHCO}_{3}$, $1.2 \mathrm{mM} \mathrm{MgCl}_{2}, 11.5 \mathrm{mM}$ glucose, with a $95 \% \mathrm{O}_{2}-5 \% \mathrm{CO}_{2}$ saturation at $37 \pm 0.5^{\circ} \mathrm{C}$ ). The bicarbonate buffer flow velocity was $10 \mathrm{~mL} / \mathrm{min}$. The heart was cut, and a stimulating electrode was sutured on each distal side under a dissecting microscope. A recording electrode was inserted until it reached the ventricular subendocardium of the septum. The reference electrode was perfused at $\sim 1 \mathrm{~mL} / \mathrm{min}$ with isotonic $\mathrm{KCl}$, enriched with $1.8 \mathrm{mM}$ $\mathrm{CaCl}_{2}$, and grounded. The standard glass microelectrode (filled with $3 \mathrm{M} \mathrm{KCl}$ solution), electron-stimulating devices (SEN-7203, Nihon Kohden, Japan), isolator (SS-202J, Nihon Kohden), and microelectrode propeller (Narishige, Japan) were prepared. The electrodes were connected using $\mathrm{Ag} / \mathrm{AgCl}$ wire to a high-input impedance buffer amplifier connected to a differential microelectrode amplifier (300 M $\Omega$ Intra767, WPI, USA). The APD determination method was analyzed in detail using Powerlab/4sp system (Australia). The ventricular APD was measured, as well as the 50 and $90 \%$ APD $\left(\right.$ APD $_{50}$ and $A P D_{90}$, respectively).

\section{Statistical analysis}

Data were analyzed using Excel and SPSS (version 11.5). A $P$ value of $<0.05$ was considered to be statistically significant. Comparison between two means was carried out using a paired Student $t$-test. Data are reported as means \pm SE, unless otherwise stated.

\section{Results}

\section{Hemodynamics and echocardiography}

The data suggest that rapid right ventricular pacing produced low-output HF in the dogs. Echocardiographic examination revealed progressive left ventricular dilatation and contractile impairment in the pacing group. The hemodynamic parameters of the pacing group are summarized in Table 1. Evaluation of cardiac hemodynamics, mass, and chamber size in rapid ventricular pacing dogs showed increased diastolic IVS thickness (baseline: $0.37 \pm 0.04 \mathrm{~cm}$; 3-week pacing: $0.46 \pm 0.07 \mathrm{~cm}$; $\mathrm{P}<0.01$ ), left ventricular posterior wall thickness diastole 
Table 1. Hemodynamic parameters in the pacing group at baseline and after 3 weeks.

\begin{tabular}{|c|c|c|}
\hline & 0 weeks & 3 weeks \\
\hline $\begin{array}{l}\text { Left ventricular ejection fraction } \\
(\%)\end{array}$ & $62.6 \pm 4.77$ & $42.4 \pm 3.21^{*}$ \\
\hline Fraction shortening (\%) & $27.4 \pm 1.95$ & $21.0 \pm 1.95^{*}$ \\
\hline $\begin{array}{l}\text { Left ventricular velocity of } \\
\text { circumference }(\%)\end{array}$ & $65.2 \pm 2.77$ & $54.0 \pm 2.92^{*}$ \\
\hline Stroke volume (L) & $5.47 \pm 0.42$ & $4.36 \pm 0.36^{*}$ \\
\hline $\begin{array}{l}\text { Left ventricular end-systolic } \\
\text { pressure }(\mathrm{kPa})\end{array}$ & $0.84 \pm 0.04$ & $0.70 \pm 0.09^{*}$ \\
\hline $\begin{array}{l}\text { Diastolic interventricular } \\
\text { septum thickness }(\mathrm{cm})\end{array}$ & $0.37 \pm 0.04$ & $0.46 \pm 0.07^{*}$ \\
\hline $\begin{array}{l}\text { Left ventricular posterior wall } \\
\text { thickness diastole }(\mathrm{cm})\end{array}$ & $0.45 \pm 0.33$ & $0.55 \pm 0.32^{*}$ \\
\hline $\begin{array}{l}\text { Left atrial systolic diameter } \\
(\mathrm{cm})\end{array}$ & $1.57 \pm 0.10$ & $2.37 \pm 0.28^{*}$ \\
\hline
\end{tabular}

Data are reported as means \pm SE. ${ }^{*} \mathrm{P}<0.01$ vs 0 weeks (Student $t$-test).

(baseline: $0.45 \pm 0.33 \mathrm{~cm}$; 3-week pacing: $0.55 \pm 0.32 \mathrm{~cm}$; $\mathrm{P}<0.01)$, and left atrial systolic diameter $(1.57 \pm 0.10$ to $2.37 \pm 0.28 \mathrm{~cm}$; all $\mathrm{P}<0.05$ vs control) during echocardiographic examination. Three-week pacing also decreased the left ventricular ejection fraction $(62.6 \pm 4.77$ to $42.4 \pm 3.21 \%)$, fraction shortening (27.4 \pm 1.95 to $21.0 \pm 1.95 \%)$, and stroke volume $(5.47 \pm 0.42$ to $4.36 \pm 0.36 \mathrm{~L}$; all $\mathrm{P}<0.01$ ) as assessed using echocardiography. These data suggest that the left and right ventricular walls became thinner, the heart chambers dilated, and the relative cardiac weight increased in the pacing group (Tables 1 and 2).

The pacing group also exhibited increased heart-weight to body-weight ratio (control: $9.7 \pm 0.44$; 3-week pacing: $12.1 \pm 1.43 \mathrm{~g} / \mathrm{kg}$ ), left ventricular diameter (control:

Table 2. Hemodynamic parameters in the control group at baseline and after 3 weeks.

\begin{tabular}{|c|c|c|}
\hline & 0 weeks & 3 weeks \\
\hline $\begin{array}{l}\text { Left ventricular ejection fraction } \\
(\%)\end{array}$ & $69.6 \pm 3.30$ & $68.0 \pm 4.20$ \\
\hline Fraction shortening (\%) & $28.2 \pm 2.0$ & $27.3 \pm 1.84$ \\
\hline $\begin{array}{l}\text { Left ventricular velocity of } \\
\text { circumference }(\%)\end{array}$ & $68.0 \pm 3.44$ & $67.7 \pm 3.82$ \\
\hline Stroke volume (L) & $6.40 \pm 0.32$ & $6.33 \pm 0.28$ \\
\hline $\begin{array}{l}\text { Left ventricular end-systolic } \\
\text { pressure }(\mathrm{kPa})\end{array}$ & $0.9 \pm 0.03$ & $1.00 \pm 0.02$ \\
\hline $\begin{array}{l}\text { Diastolic interventricular septum } \\
\text { thickness }(\mathrm{cm})\end{array}$ & $0.44 \pm 0.02$ & $0.46 \pm 0.03$ \\
\hline $\begin{array}{l}\text { Left ventricular posterior wall } \\
\text { thickness diastole }(\mathrm{cm})\end{array}$ & $0.50 \pm 0.24$ & $0.52 \pm 0.15$ \\
\hline Left atrial systolic diameter $(\mathrm{cm})$ & $2.3 \pm 0.10$ & $2.29 \pm 0.12$ \\
\hline
\end{tabular}

Data are reported as means \pm SE.
45.10 $\pm 1.92 \mathrm{~mm}$; 3-week pacing: $55.82 \pm 2.61 \mathrm{~mm}$ ), and right ventricular diameter (control: $43.12 \pm 2.10 \mathrm{~mm}$; 3-week pacing: $53.24 \pm 2.78 \mathrm{~mm}$; all $\mathrm{P}<0.01 \mathrm{vs}$ control) during postmortem examination. The 3 -week pacing group also had decreased right ventricular wall thickness $(8.08 \pm 0.85$ to $6.14 \pm 0.77 \mathrm{~mm})$ and left ventricular wall thickness $(10.62 \pm 0.68$ to $9.7 \pm 2.3 \mathrm{~mm}$; both $\mathrm{P}<0.01)$ as assessed by echocardiography (Table 3 ).

\section{Cardiac histology}

Light microscopic examination of ventricular myocytes in the pacing group showed disorganized structures: disrupted myocardial transverse striations, wave-like arrangements of cardiac muscle fibers, dissolved and necrotic cell cytoplasm, mesenchymal blood vessel dilatation and congestion, erythrocyte leakage, and small inflammatory cell infiltration. In contrast, no such changes occurred in the control group (Figure 1). No abnormalities were observed following electron microscopy studies of the biopsy specimens from the control group. Electron microscopy of ventricular myocytes from the pacing group showed disorganized structures such as myocardial fiber hypertrophy, disordered myofilaments, edema, damaged striated muscle framework, disrupted myocardial fibers, unclear $Z$ lines, disrupted and engorged mitochondrial membranes, rarefaction of cristae, vacuolization, distended sarcoplasmic reticulum, and intercalary disc abnormalities. Once again, no such changes occurred in the control group. Distinct mitochondrial swelling was observed in the remaining three specimens, associated with decreased density and definition of cristae, which is consistent with lysis of cristae. Abnormalities were confined to the mitochondria without evidence of intracellular swelling, swelling of the transverse tubular system or of the sarcoplasmic reticulum (Figure 2). Light microscopy of the biopsy specimens, showed that addition of $\mathrm{Ca}^{2+}$ led to an abrupt decrease in light absorbance (as a result of increased light scattering), indicating mitochondrial swelling.

Table 3. Heart mass and dimensions in control and pacing groups.

\begin{tabular}{lcc}
\hline & Control & Post-pacing \\
\hline $\begin{array}{l}\text { Heart weight/body weight } \\
(\mathrm{g} / \mathrm{kg})\end{array}$ & $9.7 \pm 0.44$ & $12.1 \pm 1.43^{*}$ \\
$\begin{array}{l}\text { Right ventricular wall thickness } \\
(\mathrm{mm})\end{array}$ & $8.08 \pm 0.85$ & $6.14 \pm 0.77^{*}$ \\
$\begin{array}{l}\text { Left ventricular wall thickness } \\
(\mathrm{mm})\end{array}$ & $10.62 \pm 0.68$ & $9.7 \pm 2.30^{*}$ \\
$\begin{array}{l}\text { Ventricular interval thickness } \\
(\mathrm{mm})\end{array}$ & $7.46 \pm 0.57$ & $5.62 \pm 0.34^{*}$ \\
$\begin{array}{l}\text { Left ventricular diameter }(\mathrm{mm}) \\
\begin{array}{l}\text { Right ventricular diameter } \\
(\mathrm{mm})\end{array}\end{array}$ & $45.10 \pm 1.92$ & $55.82 \pm 2.61^{*}$ \\
\hline
\end{tabular}

Data are reported as means $\pm \mathrm{SE}$. ${ }^{*} \mathrm{P}<0.01$ vs control group (Student $t$-test). 


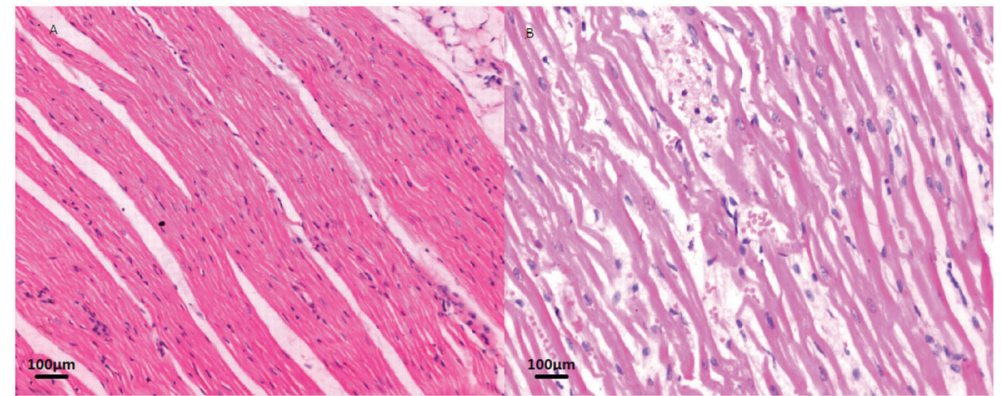

Figure 1. Representative heart sections from control $(A)$ and pacing $(B)$ dogs obtained by light microscopy. Panel $B$ shows disorganized structures: disrupted myocardial transverse striations, wave-like arrangements of cardiac muscle fibers, dissolved and necrotic cell cytoplasm, mesenchymal blood vessel dilatation and congestion, erythrocyte leakage, and small inflammatory cell infiltration, which did not occur in the control group.

\section{Action potential duration}

Differences in myocyte action potential shape and duration were observed in dogs in both the control and pacing groups. Representative action potentials recorded at $37^{\circ} \mathrm{C}$ in control and pacing dogs are shown in Figure 3. The action potentials recorded from myocytes in failing ventricles were significantly prolonged compared to the control myocytes, with decreased notch amplitude in phase 1 and increased plateau height following the notch. The resting membrane potential was unchanged. The action potential characteristics of the two groups are summarized in Table 4.

\section{Discussion}

The major new finding of the present study is that myocardial remodeling, as well as bioelectric changes in tachycardia-induced $\mathrm{HF}$, support an important role of remodeling in HF progression (17). Several possible explanations can account for the observed results in myocardial electrical and mechanical remodeling (4-9). The dogs subjected to rapid pacing developed persistent tachycardia, which resulted in low-output HF characterized by biventricular pump dysfunction, biventricular cardiac dilation, and non-ischemic impairment of left ventricular contractility (4-9). There was direct evidence of the effects of short-term (3 weeks) myocardial remodeling in dogs with an increase in tachycardia-induced HF. Shimizu et al. (18) reported data on the interaction between three types of cardiac remodeling, namely, structural (hypertrophy), contractile (decreased or increased contraction), and electrical (altered action potential profile). Schreiner et al. (19) presented a well-documented study on remodeling in a dog with chronic AV block, which developed cardiac hypertrophy, increased contractility, and electrical remodeling with increased APD, QT dispersion, and pro-arrhythmia. Vos et al. (20) investigated how pacing would reverse these changes. Surprisingly, the parameters changed independently. Consistent with previous reports, Plotnikov (21) reviewed the body of work on the effects of pacing on normal heart electrophysiology in a model of pacing-induced HF (22). Pacing from a ventricular site and the resulting altered activation patterns of the ventricles lead to unphysiological contraction patterns and stretching of myocytes $(3,8,11-14)$. Independent studies have indicated that stretch alters protein expression. Alterations in protein expression during pacing lead to electrical remodeling of myocytes with increased APD. Several ion channels are involved, such as the transient outward $\mathrm{K}^{+}$channels, $\mathrm{Ca}^{2+}$ channels, and connexins $(10,23)$. Important findings of the present study include the differences in the action potential shape and duration

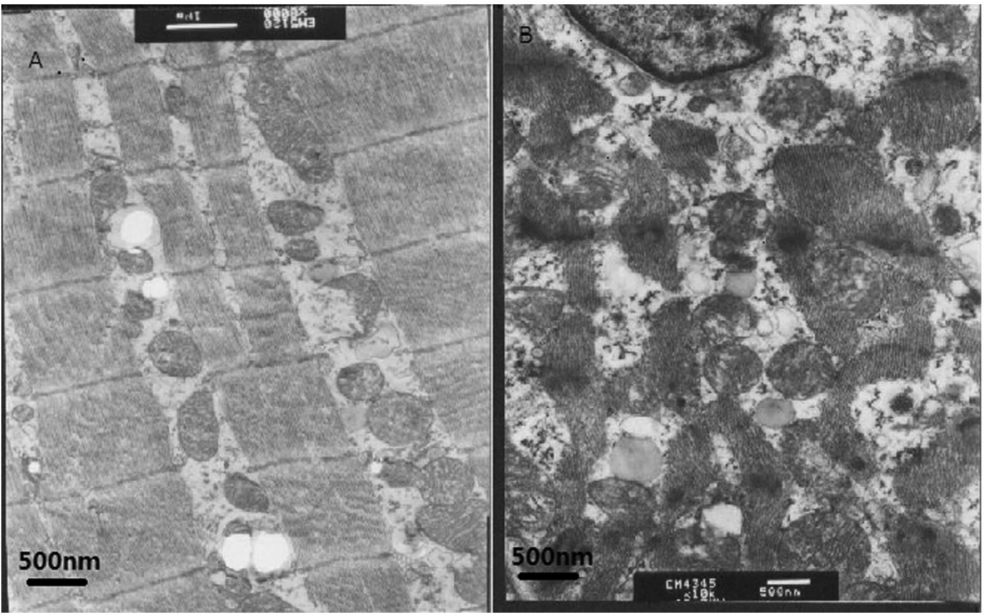

Figure 2. Representative photomicrography of ventricular myocardium from control $(A)$ and pacing $(B)$ dogs obtained by electron microscopy. Panel $B$ shows disorganized structures: myocardial fiber hypertrophy, disordered myofilaments, edema, damaged striated muscle framework, disrupted myocardial fibers, unclear Z lines, disrupted and engorged mitochondrial membranes, rarefaction of cristae, vacuolization, distended sarcoplasmic reticulum, and intercalary disc abnormalities, which did not occur in the control group. 


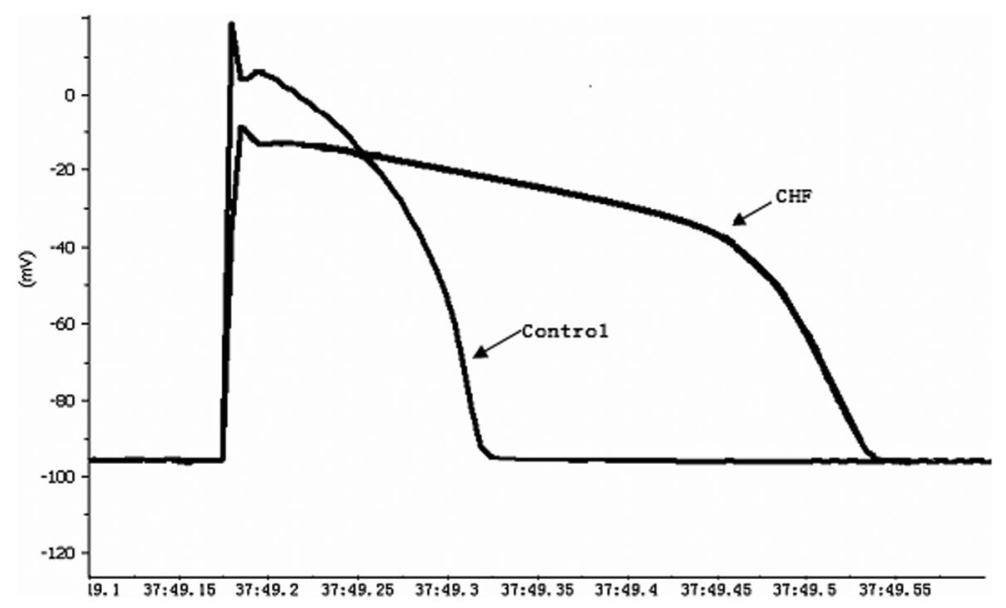

Figure 3. Representative recordings from control and pacing dogs showing action potential shape and duration. CHF: congestive heart failure.

Table 4. Bioelectric properties of hearts in control and pacing groups.

\begin{tabular}{lcc}
\hline & Control & Pacing \\
\hline APA $(m V)$ & $111.8 \pm 6.75$ & $93.8 \pm 5.17^{*}$ \\
RP $(m V)$ & $93.8 \pm 2.22$ & $93.2 \pm 2.40$ \\
APD $_{50}(\mathrm{~ms})$ & $154.0 \pm 40.37$ & $365.6 \pm 39.8^{*}$ \\
APD $_{90}(\mathrm{~ms})$ & $267.6 \pm 17.6$ & $443.0 \pm 60.17^{*}$ \\
\hline
\end{tabular}

Data are reported as means $\pm \mathrm{SE}$. APA: action potential amplitude; RP: resting potential; $\mathrm{APD}_{50}: 50 \%$ action potential duration; $\mathrm{APD}_{90}$ : $90 \%$ action potential duration. ${ }^{*} \mathrm{P}<0.05$ vs control group (Student $t$-test).

observed in this dog model of HF. According to Hopenfeld (17) and Shimizu et al. (18), the action potentials recorded from failing ventricle myocytes were significantly prolonged compared to those of controls, but the resting membrane potential was unchanged. APD prolongation is considered a major anti-arrhythmic mechanism that frequently, and paradoxically, causes arrhythmia (torsade de pointes) $(17,18)$.

Another important finding is that the dogs subjected to rapid pacing experienced not only myocardial electrical changes but also myocardial remodeling (4-9) in tachycardia-induced HF. We also described the

\section{References}

1. Nakamura R, Egashira $\mathrm{K}$, Machida $\mathrm{Y}$, Hayashidani $\mathrm{S}$, Takeya M, Utsumi $\mathrm{H}$, et al. Probucol attenuates left ventricular dysfunction and remodeling in tachycardiainduced heart failure: roles of oxidative stress and inflammation. Circulation 2002; 106: 362-367, doi: 10.1161/01.CIR. 0000021430.04195 .51$.

2. Nam MC, Aravind A, Chan K, James S, Brown N. Tachycardia-induced cardiomyopathy - a fully reversible phenomenon. Am Heart Hosp J 2010; 8: E115-E117.

3. Chen X, Sala-Mercado JA, Hammond RL, Ichinose M, histological changes that occurred in ventricular myopathy seen using light microscopy and the mitochondrial swelling and degenerated cristae seen using electron microscopy. An increase in the number of mitochondria was noted, as well as disrupted sarcoplasmic reticulum, enlarged nuclei, and dilated rough endoplasmic reticulum. A review of studies on remodeling showed that although alterations of these cytoplasmic structures varied, an increase in APD was a remarkably consistent finding.

Our data support the hypothesis that ventricular pacing may induce electrical remodeling. There is also evidence (17) of structural remodeling and, to a lesser extent, electrical remodeling in patients with underlying heart disease and remodeling at baseline. In summary, myocardial electrical and structural remodeling play an essential role in the progression of congestive HF in tachycardia-induced processes. Further studies are needed to determine the beneficial effects of pacing therapy. Adverse effects need thorough investigation and must be evaluated in light of other positive effects of pacing therapy.

\section{Acknowledgments}

Research supported by the Anhui Provincial University Scientific Research Project (\#Kj2011z213).
Soltani S, Mukkamala R, et al. Dynamic control of maximal ventricular elastance via the baroreflex and force-frequency relation in awake dogs before and after pacing-induced heart failure. Am J Physiol Heart Circ Physiol 2010; 299: H62-H69, doi: 10.1152/ajpheart.00922.2009.

4. Konstam MA, Kramer DG, Patel AR, Maron MS, Udelson JE. Left ventricular remodeling in heart failure: current concepts in clinical significance and assessment. JACC Cardiovasc Imaging 2011; 4: 98-108, doi: 10.1016/j.jcmg.2010.10.008.

5. Cohn JN, Ferrari R, Sharpe N. Cardiac remodeling - concepts 
and clinical implications: a consensus paper from an international forum on cardiac remodeling. J Am Coll Cardiol 2000; 35: 569-582, doi: 10.1016/S0735-1097(99)00630-0.

6. Swynghedauw B. Molecular mechanisms of myocardial remodeling. Physiol Rev 1999; 79: 215-262.

7. McElmurray JH III, Mukherjee R, New RB, Sampson AC, King MK, Hendrick JW, et al. Angiotensin-converting enzyme and matrix metalloproteinase inhibition with developing heart failure: comparative effects on left ventricular function and geometry. J Pharmacol Exp Ther 1999; 291: 799-811.

8. Su JB, Barbe F, Laplace M, Crozatier B, Hittinger L. Regional alterations of left ventricular contraction and inotropic reserve in conscious dogs with heart failure. Cardiovasc Res 1995; 30: 848-856.

9. Dastidar AG, Sheridan P. Tachycardia-induced cardiomyopathy caused by a special pacemaker algorithm. Cardiol J 2011; 18: 573-576, doi: 10.5603/CJ.2011.0018.

10. Hill JA. Electrical remodeling in cardiac hypertrophy. Trends Cardiovasc Med 2003; 13: 316-322, doi: 10.1016/j.tcm. 2003.08.002.

11. Power JM, Tonkin AM. Large animal models of heart failure. Aust N Z J Med 1999; 29: 395-402, doi: 10.1111/j.14455994.1999.tb00734.x.

12. Paslawska U, Gajek J, Kiczak L, Noszczyk-Nowak A, Skrzypczak P, Bania J, et al. Development of porcine model of chronic tachycardia-induced cardiomyopathy. Int J Cardiol 2011; 153: 36-41, doi: 10.1016/j.ijcard.2010.08.033.

13. Umana E, Solares CA, Alpert MA. Tachycardia-induced cardiomyopathy. Am J Med 2003; 114: 51-55, doi: 10.1016/ S0002-9343(02)01472-9.

14. Gao Z, Xu H, DiSilvestre D, Halperin VL, Tunin R, Tian Y, et al. Transcriptomic profiling of the canine tachycardiainduced heart failure model: global comparison to human and murine heart failure. J Mol Cell Cardiol 2006; 40: 76-86, doi: 10.1016/j.yjmcc.2005.08.002.

15. McMahon WS, Mukherjee R, Gillette PC, Crawford FA, Spinale FG. Right and left ventricular geometry and myocyte contractile processes with dilated cardiomyopathy: myocyte growth and beta-adrenergic responsiveness. Cardiovasc Res 1996; 31: 314-323.
16. Li D, Shinagawa K, Pang L, Leung TK, Cardin S, Wang Z, et al. Effects of angiotensin-converting enzyme inhibition on the development of the atrial fibrillation substrate in dogs with ventricular tachypacing-induced congestive heart failure. Circulation 2001; 104: 2608-2614, doi: 10.1161/ hc4601.099402

17. Hopenfeld B. Mechanism for action potential alternans: the interplay between L-type calcium current and transient outward current. Heart Rhythm 2006; 3: 345-352, doi: 10.1016/j.hrthm.2005.11.016.

18. Shimizu H, Inoue T, Miwa S, Yoshida A, Itagaki T, Ohnishi $Y$, et al. Dispersion of the monophasic action potential duration in patients with polymorphic ventricular tachycardia. J Electrocardiol 1999; 32: 217-224, doi: 10.1016/S00220736(99)90104-8.

19. Schreiner KD, Voss F, Senges JC, Becker R, Kraft P, Bauer $A$, et al. Tridimensional activation patterns of acquired torsade-de-pointes tachycardias in dogs with chronic AVblock. Basic Res Cardiol 2004; 99: 288-298, doi: 10.1007/ s00395-004-0469-4.

20. Vos MA, de Groot SH, Verduyn SC, van der Zande J, Leunissen HD, Cleutjens JP, et al. Enhanced susceptibility for acquired torsade de pointes arrhythmias in the dog with chronic, complete AV block is related to cardiac hypertrophy and electrical remodeling. Circulation 1998; 98: 1125-1135, doi: 10.1161/01.CIR.98.11.1125.

21. Plotnikov AN, Yu H, Geller JC, Gainullin RZ, Chandra P, Patberg KW, et al. Role of L-type calcium channels in pacing-induced short-term and long-term cardiac memory in canine heart. Circulation 2003; 107: 2844-2849, doi: 10.1161/01.CIR.0000068376.88600.41.

22. Teramura S, Yamakado T, Maeda M, Nakano T. Effects of $\mathrm{MCl}-154$, a calcium sensitizer, on left ventricular systolic and diastolic function in pacing-induced heart failure in the dog. Circulation 1997; 95: 732-739, doi: 10.1161/01.CIR. 95.3.732.

23. Toyama J, Kamiya K, Cheng J, Lee JK, Suzuki R, Kodama I. Vesnarinone prolongs action potential duration without reverse frequency dependence in rabbit ventricular muscle by blocking the delayed rectifier $\mathrm{K}^{+}$current. Circulation 1997; 96: 3696-3703, doi: 10.1161/01.CIR.96.10.3696. 